\title{
La gestión del conocimiento basado en la teoría de Nonaka y Takeuchi
}

\section{Knowledge management based on the theory of Nonaka and Takeuchi}

Ing. Ruth Sabrina Rojas Dávila

Universidad Católica de Santiago de Guayaquil, Ecuador

Ing. Carlos Luis Torres Briones

Universidad de Guayaquil, Ecuador

Autor por Correspondencia: ruth.rojas@cu.ucsg.edu.ec, carlos.torresb@ug.edu.ec

Fecha de recepción: 25 de Enero de 2017 - Fecha de aceptación: 25 de Marzo de 2017

Resumen: El conocimiento está traspasando las fronteras de la economía como parte de un recurso vital para las economías del mundo que se transforma en una estrategia que genera una creación de ideas para las organizaciones, para el ser humano y para el sentido relacional. (Meyer \& Sugiyama, 2006). Es importante recalcar que el desarrollo del capital humano, las organizaciones, ponen en práctica la gestión de recursos humanos, sobre el desarrollo de líderes transformacionales y esto a su vez en la aplicación de la gestión del conocimiento. El conocimiento o la gestión cognitiva, así como el empoderamiento administrativo son conceptos que han venido aplicándose a la gestión educativa, en las últimas décadas. Esto dio lugar a la aparición de muchas prácticas, que empezaron a diferir de cada una de las especializaciones de los investigadores y sus puntos de vista. Estos conceptos están todavía en fase de desarrollo y exploración. La aplicación del método de la gestión del conocimiento y el empoderamiento administrativo de los trabajadores en cualquier institución parece proporcionar nuevas habilidades $\mathrm{y}$ distintas posibilidades competitivas.

Palabras claves: gestión del conocimiento; conocimiento organizacional; creación de conocimiento

\begin{abstract}
Knowledge is crossing the frontiers of the economy as part of a vital resource for the economies of the world that is transformed into a strategy that generates a creation of ideas for organizations, for the human being and for the relational sense. (Meyer \& Sugiyama, 2006). It is important to emphasize that the development of human capital, the organizations, put into practice human resources management, the development of transformational leaders and this in turn in the application of knowledge management. Knowledge or cognitive management, as well as administrative empowerment are concepts that have been applied to educational management in recent decades. This led to the emergence of many practices, which began to differ from each of the researchers' specializations and their points of view. These concepts are still under development and exploration. The application of the method of knowledge management and the administrative empowerment of workers in any institution seems to provide new skills and different competitive possibilities.
\end{abstract}

Key Words: knowledge management; organizational knowledge; knowledge creation 


\section{Introducción}

La aparición y expansión de gestión del conocimiento y la gestión de la información, por una parte, está en deuda con el estudio y la investigación en el ámbito del trabajo y la gestión empresarial, por otro lado el resultado de estas tendencias organizacionales que son pioneras en el establecimiento de los sistemas de gestión de conocimiento y en su intento por generar una modificación y adaptación de esta práctica en las condiciones del entorno interno de la organización. (Goudarzvandchigini, 2011).

El desarrollo de las competencias de los recursos genera el establecimiento de una actividad considerada primordial para cualquier tipo de organización, esto a su vez genera enormes esfuerzos para superar a sus competidores no solo en calidad sino en servicio al cliente, así como el desarrollo de nuevos productos. La presente revisión de literatura busca identificar como la teoría de la gestión del conocimiento se encuentra alineada a las organizaciones de cualquier tipo, se busca investigar y analizar las estrategias que se tomaron para llegar a la aplicación de la teorías, así como las acciones ejecutivas que hacen que la gestión del conocimiento y liderazgo transformacional en la organización sea considerada una estrategia exitosa empresarialmente.

\section{Marco Teórico y conceptual}

Según (Nonaka \& Takeuchi, 1995) proponen una Teoría para explicar el fenómeno de la creación de conocimiento organizacional, cuyo conocimiento lo definen como "creencia verdadera justificada" (p.21) para reflejar el conocimiento actual en el que se enmarca la existencia del mismo. Esta creación de conocimiento organizacional se definió como "... la capacidad de una empresa en su conjunto para crear nuevos conocimientos, así como difundirlo en toda la organización y que queden establecidos en productos, servicios y sistemas "(p. 3).

Los mismos autores inician su argumento estableciendo que el conocimiento es creado inicialmente por los individuos dentro de las organizaciones y que el mismo se convierte en conocimiento organizacional, descrito a través de un proceso establecido por la teoría.

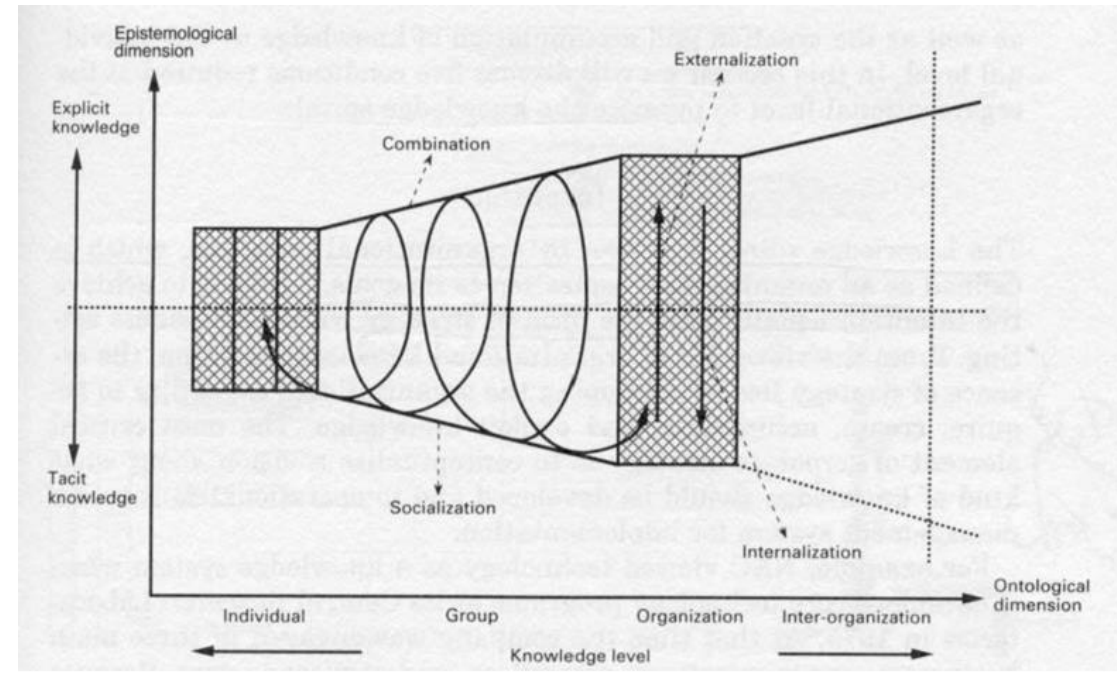

Figura 1 Espiral del conocimiento organizacional 
Nota: Tomado de Nonaka \& Takeuchi "The Knowledge creating company", 1995.

(McLean, 2004) Se describieron dos dimensiones para la creación del conocimiento organizacional, una es la epistemológica y la otra la ontológica. En lo epistemológico los autores Nonaka \& Takeuchi (1995), reconocen dos tipos de conocimiento: tácito y explícito. El conocimiento explícito es el conocimiento que pueden ser escritos y transferidos con relativa facilidad de una persona a otra. Por otro lado el conocimiento tácito, es más difícil de articular porque a menudo surge por parte de la experiencia.

La dimensión ontológica se extiende a partir del conocimiento del individuo en un extremo y es transferida de allí al resto del equipo, grupo, organización y más allá. "El espiral emerge cuando la interacción entre el conocimiento tácito y explícito se eleva dinámicamente desde la parte ontológica a los niveles más altos dentro de las organizaciones"(Nonaka y Takeuchi, 1995, pg. 57).

(Nonaka, 1994) A pesar de que el conocimiento es un concepto multifacético la historia en su parte filosófica nos muestra desde el periodo Griego clásico como la búsqueda interminable de información nos lleva hacia un significado del verdadero conocimiento. En la epistemología tradicional se adopta una definición de conocimiento como "creencia verdadera justificada", el autor relata que si bien los argumentos de la epistemología tradicional se centran en "veracidad" como el atributo esencial del conocimiento, también forma parte importante considerar el conocimiento como una creencia personal que se destaca partiendo de la importancia en la justificación de conocimientos.

Desde el punto de vista de los conocimientos tradicionales y de la epistemología, la teoría de la creación de conocimiento hace hincapié en la forma absoluta, estática y no humana naturaleza del conocimiento, que normalmente se expresa en formas proposicional lógica, este último considera el conocimiento como un proceso humano dinámico para justificar las creencias personales como parte de una aspiración a la verdad que maneja cada uno de los individuos.

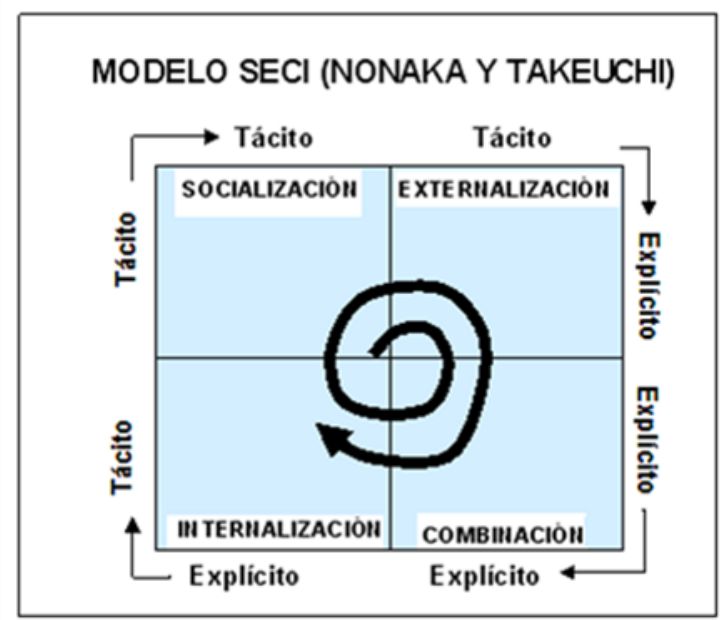

Figura 2 Modos para la creación del conocimiento

Nota: Tomado de I, Nonaka "Dynamic Theory of Organizational Knowledge Creation", 1994

De acuerdo a los descrito por Nonaka, (1994), existen cuatro modos de conversión del conocimiento, esto se da a través de una conversión entre el conocimiento tácito y el explícito 
que permite postular diferentes modos de conversión del conocimiento: (1) de conocimiento tácito al conocimiento tácito, (2) del conocimiento explícito al conocimiento explícito, (3) del conocimiento tácito al conocimiento explícito, y (4) del conocimiento explícito al conocimiento tácito.

En el primer caso existe un modo de conversión del conocimiento que nos permite transformar el conocimiento tácito a través de la interacción entre los individuos, se debe considerar algo importante de ser analizado y es que el individuo dentro de la organización puede adquirir un conocimiento tácito sin lenguaje, un ejemplo que se aplica es el de los aprendices que trabajan con sus mentores y estos a su vez aprenden a elaborar artesanías no a través del lenguaje, sino por la observación, la imitación y la práctica. En un entorno comercial, la formación en el puesto de trabajo utiliza este mismo principio. La clave para la adquisición de conocimiento tácito es la experiencia. Si no existe la experiencia compartida, es extremadamente difícil para las personas que compartan entre otros procesos de pensamiento. Este proceso de creación de conocimiento tácito a través de compartir experiencia se lo conoce como socialización.

El segundo modo de conocimiento, la conversión implica la utilización de procesos sociales para combinar distintos análisis de conocimiento explícito celebrada por los individuos.

Las personas buscan intercambiar y combinar conocimiento a través de los mecanismos como reuniones y conversaciones telefónicas. La reconfiguración de la información existente a través de la clasificación, adición y re contextualización y re categorización de conocimiento explícito puede conducir hacia nuevos conocimientos. Los modernos sistemas informáticos proporcionan un ejemplo claro de lo que se busca en este proceso de crear conocimiento explícito del conocimiento y este se transforma en combinación.

El tercero y cuarto modo de conversión de conocimiento se refieren a patrones de conversión que involucra tanto el conocimiento tácito y explícito. Estos modos de conversión buscan captar la idea de que el conocimiento tácito y explícito se complementa y pueden expandirse a lo largo del tiempo a través de un proceso de interacción mutua. Esta interacción involucra dos operaciones diferentes. Uno de ellos es la conversión del conocimiento tácito en conocimiento explícito, que se llamará la externalización, y la otra es la conversión de conocimiento explícito en conocimiento tácito, que tiene cierta similitud con el concepto tradicional de aprendizaje y que se lo conoce como internalización.

Tres de los cuatro tipos de conocimiento de conversión de socialización, combinación e internalización, tienen parcialmente análogos con aspectos de la teoría organizacional. Un ejemplo claro de esto es la socialización que se encuentra conectada a las teorías de la cultura organizacional, mientras que la combinación de todas está relacionada con en el procesamiento de la información y la internalización que tiene asociaciones con el aprendizaje organizacional. (Nonaka, 1994)

\section{Materiales y métodos}

Para evaluar la validez de la teoría de la gestión del conocimiento se realizó una revisión literaria de la investigación titulada "Una encuesta de la relación entre liderazgo 
transformacional, aprendizaje organizacional, gestión del conocimiento, innovación organizacional y el desempeño Organizacional", la finalidad de revisar esta investigación es identificar la intención que existe entre el liderazgo transformacional, el aprendizaje organizacional, y la teoría de la gestión del conocimiento, junto con la innovación organizativa y el desempeño. (Gelard, Boroumand, \& Mohammadi, 2014).

También se revisó un estudio titulado "Liderazgo Transformacional y los beneficios en el capital humano", la herramienta utilizada fue una encuesta que medía como la gestión del conocimiento establece una relación entre el liderazgo transformacional, el conocimiento y la percepción de los empleados como capital humano en el proceso de gestión y construcción del desarrollo de la cultura organizativa para fomentar la participación activa del personal. (Gelard, Boroumand, \& Mohammadi, 2014).

En otro estudio realizado se demostró a través de una encuesta la relación entre los estilos de liderazgo y el intercambio de conocimientos: un estudio de caso en empresas de servicios, miembros de alianzas estratégicas, se intenta descubrir la relación entre liderazgo transformacional y liderazgo transaccional con el intercambio de conocimientos. (Chen, 2006)

Adicional a esto se realizó un levantamiento de información a través de journals con reconocimiento académico, así como la búsqueda de libros que validen el uso de la teoría.

\section{Análisis}

Este método busca aprovechar un sistema preciso de gestión y práctica que sea adaptable a los distintos procesos ocupacionales. Además se comprende que una parte complementaria e integradora de la gestión del conocimiento busca enmarcarse en el desarrollo de la innovación. (Badah, 2011).

En la era del conocimiento, éste termina siendo el capital más importante de las organizaciones que buscan generar una ventaja competitiva sostenible. Una organización puede lograr conocimiento y experiencias con la ayuda de una gestión sistemática y global y puede utilizarlas para proteger su éxito y competitividad en el largo plazo (Monavarian y Asgari, 2009).

El éxito de la organización ha sido ampliamente confirmado (Chennemaneni, 2008), a través del uso de la gestión del conocimiento que busca crear y desarrollar las propiedades de conocimiento dentro de las organizaciones. Esta gestión del conocimiento se refiere a los esfuerzos que la teoría establece de forma sistemática con la finalidad de encontrar, crear, hacer accesible, aplicar las capitales intangibles de la organización y fortalecer la cultura del aprendizaje continuo y el intercambio de conocimientos en la organización (Monavarian \& Asgari, 2009).

La gestión del conocimiento es un proceso complejo y su implementación requiere la aplicación, así como el uso eficaz de una comprensión precisa y clara de factores que pueden afectar su proceso de implementación. Estos factores en su conjunto se consideran facilitadores para comprender el funcionamiento de la gestión del conocimiento. (Rahnavard \& Mohammadi, 2009). 
El carácter humano-social de las medidas de gestión del conocimiento, hace que el papel de una participación activa y entusiasta del factor humano en estas medidas sea necesario según el estilo de liderazgo que maneje y como ejerza la influencia en la creación de motivación, participación y compromiso, esto a su vez se aproxima a lo descrito en el concepto de liderazgo transformacional y que a futuro puede tener un efecto considerable en la capacidad y disposición de las personas a participar en las medidas de gestión del conocimiento. (Yaghoubi, Mahallati, Safari, \& Ali, 2014)

\section{Discusión de resultados}

En los últimos años la teoría de la gestión del conocimiento ha evolucionado de acuerdo a las condiciones cambiantes de la naturaleza de cada uno de los negocios, lo que lo convierte en una estrategia de capital humano capaz de adaptarse a factores positivos y negativos que aquejan en las organizaciones.

La organización que tienen un participación activa en la gestión del conocimiento establecen sistemas de comunicación modernos que se han convertido en un entorno social diseñado para especialistas dentro de estructuras organizacionales adecuadas, en donde la satisfacción de las necesidades del mercado se vuelve más eficiente.

Algunos de los autores aclaran que tan importante se vuelve la inclusión del trabajador en esta nueva era del conocimiento, en las cuales se establecen nuevas herramientas que generan una producción activa en el reconocimiento de las necesidades de información tanto interna como externamente.

La aparición de una sociedad basada en el conocimiento ha llegado y las organizaciones que pueden tener éxito en la sociedad global de la información son aquellas que pueden identificar, valorar, crear y desarrollar sus conocimientos. Esto se demuestra con el cambio y la innovación que se transforman en competencias centrales y que buscan ser dominadas para que las organizaciones tengan éxito, que requieren en cada una de ellas. (Crawford, 2006)

\section{Conclusiones}

La gestión del conocimiento ha aumentado en popularidad y credibilidad como herramienta de gestión y disciplina de investigación, existe una fuerte preocupación sobre si la gestión del conocimiento es simplemente una moda pasajera, de los investigadores y académicos que han buscado debatir sobre sus características o se mantiene a lo largo del tiempo con políticas clara para ser estudiadas en los diferentes entornos.

Algunos autores han adoptado la opinión de que la gestión del conocimiento que ciertamente no es una moda por diferentes razones, y de acuerdo con la opinión de que estas razones forman parte de la economía del conocimiento (Stankosky, 2005).

Existe también otra opinión que resalta que los investigadores han tomado el concepto de gestión del conocimiento hacia la búsqueda de la información basada en características sofisticadas, donde existen hallazgos que sugieren que la gestión del conocimiento se encuentra 
en un proceso de establecer la aplicación de las diferentes teorías dentro de las organizaciones, lo que genera ventajas competitivas que empiezan a ganar credibilidad y no solo se aplica a nivel empresarial sino en proyectos de investigación, de aquí nacen algunas preguntas que ha futuro se generan a través de las unidades educativas, que son los principales motores para establecer esta teoría, en donde la gestión del conocimiento busca establecer investigaciones que validen su uso, basándonos en esto ¿está preparada la educación superior para la gestión del conocimiento en la Administración?". (Joy \& Taylor, 2008)

En la búsqueda del conocimiento y bajo esta perspectiva, las organizaciones industriales que mantienen un proceso de aprendizaje llegan a mejorar el conocimiento disponible para la organización y para amplificar el valor de sus activos intelectuales, esto de la mano con el capital de innovación cuando se aplica a cualquier tipo de organizaciones. Se dice que si una organización demuestra competencia en la gestión del conocimiento se vuelven más innovadores y generadores de ideas creativas dentro del entorno empresarial.

Para Salisbury (2003), la gestión del conocimiento se define como el despliegue de un sistema integral que realza el crecimiento del conocimiento de una organización. Este despliegue se mide con el esfuerzo para que la gestión del conocimiento dentro de la organización, así como el uso del conocimiento de una manera efectiva y eficiente para el beneficio de la misma a largo plazo. Y es que la eficacia de la gestión del conocimiento se considera también como una disciplina que busca estar centrada en el desarrollo para el logro de los objetivos estratégicos del negocio. Adicional a esto, el conocimiento puede analizarse desde una perspectiva de proceso, esto descrito por (Gold, Malhotra \& Segars, 2001; Zheng, 2005) en donde se puede concebir que la eficacia de una organización busca gestión de los conocimientos adquiridos, compartidos y aplicados por sus empleados a su vez un mejoramiento dentro de la aplicación del conocimiento en la innovación organizacional para mejorar la actuación dentro de las organizaciones y el mejoramiento del desempeño de la innovación como paso para el logro de una ventaja competitiva. (Ling \& Mohd, 2011).

Al concentrarse en el concepto de la creación de conocimiento organizacional, ha sido posible desarrollar una perspectiva que va más allá de la simple noción de aprendizaje organizacional. En el lenguaje de la discusión actual, el aprendizaje puede estar relacionado a la internalización, que no es sino uno de los cuatro modos de conversión necesaria para crear nuevo conocimiento organizacional, que en muchas ocasiones es más amplio y concepto dinámico.

Se han ofrecido algunas propuestas prácticas para la aplicación más eficaz de la creación de conocimiento basados en esta teoría, y a su vez analizar como emerge la organización a través de una característica cada vez más importante dentro del desarrollo industrial avanzado, para esto es necesario prestar mayor atención a los procesos por los cuales se crea y se evalúa la calidad y valor tanto para la organización y la sociedad. Si las organizaciones de hoy no son capaces de adaptarse a los cambios que se generan dentro del entorno, estos perderán la competencia y terminarán rezagados con un manejo de conocimiento mediocre. En tal condición, las organizaciones necesitan líderes como lo demuestran los estudios, que sean capaces de convertir sus empresas en organizaciones capaces de progresar e incluso de sobrevivir en situaciones adversas. 
Se analizó la incidencia de los líderes transformacionales y como estos guían la organización desde el presente hacia el futuro y como han generado nuevos objetivos y metas que transforman a una empresa en la generadora de valor dentro de un mercado tan dinámico y cambiante. (Gelard, Boroumand, \& Mohammadi, 2014).

\section{Bibliografía}

Badah, A. (2011). Relationship between the knowledge management processes and the administrative empowerment with the employees of the ministry of higher education and scientific research- Jordan. European Scientific Journal, 8 (28), 191-209.

Chen, L. (2006). Relationship between leadership behaviors and knowledge sharing in professional service firms engaged in strategic alliances. Journal of Applied Management and Entrepreneurship, 11 (2), 51-70.

Crawford, C. (2006). Exploring the relationship between knowledge management and transformational leadership. Knowledge Management and Leadership, 7 (8).

Gelard, P., Boroumand, Z., \& Mohammadi, A. (2014). Relationship between Transformational Leadership and Knowledge Management. International Journal of Information Science and Management, 12 (2), 67-82.

Goudarzvandchigini, M. (2011). Knowledge Management and Transformational Leadership in Organizational Success. International Journal of Information and Education Technology, 1 (5), 377-379.

Joy, D., \& Taylor, J. (2008). Knowledge Management and Higher Education: A UK Case Study. Journal of Knowledge Management, 6 (2), 85-100.

Ling, C., \& Mohd, A. (2011). Human Resource Management Practices and Organizational Innovation: Assessing the Mediating Role of Knowledge Management Effectiveness. Electronic Journal of Knowledge Management, 9 (2), 155-167.

McLean, L. (2004). A review and critique of Nonaka and Takeuchi Theory of organizational knowledge creation. Proceedings of the fifth Ireland Conference. USA.

Meyer, B., \& Sugiyama, K. (2006). The concept of knowledge in KM: A dimensional model. Journal of Knowledge Management, 10 (6), 1-22.

Nonaka, I. (1994). A Dynamic Theory of Organizational Knowledge Creation. Institute for operations research ant the management sciences, 5 (1), 14-37.

Nonaka, I., \& Takeuchi, H. (1995). The knowledge creating company. New York: Oxford University Press.

Yaghoubi, H., Mahallati, T., Safari, A., \& Ali, M. (2014). Transformational Leadership: Enabling Factor of Knowledge Management Practices. Journal of Management and Sustainability, 4 (3), 165-174. 